\title{
BIFURCATIONS OF THE FORCED VAN DER POL OSCILLATOR*
}

\author{
BY \\ P. J. HOLMES (Cornell University) \\ AND \\ D. A. RAND (University of Warwick)
}

\begin{abstract}
We discuss the bifurcations of the variational equation of the forced van der Pol oscillator and prove the existence of bifurcations of saddle connection type as postulated by M. L. Cartwright [4] and A. W. Gillies [5].
\end{abstract}

1. Introduction. The parametrized differential equation

$$
\begin{gathered}
\dot{x}_{1}=-\omega x_{2}+x_{1}\left(1-x_{1}{ }^{2}-x_{2}^{2}\right) \\
\dot{x}_{2}=\omega x_{1}+x_{2}\left(1-x_{1}{ }^{2}-x_{2}{ }^{2}\right)-F
\end{gathered}
$$

$(\omega, F>0)$, which may also be written as the vector field $X$ :

$$
X_{(\omega, F)}=\left(-\omega x_{2}+x_{1}\left(1-x_{1}{ }^{2}-x_{2}^{2}\right)\right) \frac{\partial}{\partial x_{1}}+\left(\omega x_{1}+x_{2}\left(1-x_{1}{ }^{2}+x_{2}{ }^{2}\right)-F\right) \frac{\partial}{\partial x_{2}},
$$

is the variational equation [4] of the forced van der Pol oscillator;

$$
\ddot{x}-\alpha \dot{x}+\gamma x^{2} \dot{x}+x=\Phi \sin \Omega t .
$$

Here $\alpha, \gamma$ are fixed positive small constants, $\omega=\left(1-\Omega^{2}\right) / 2 \Omega, F=\Phi / 2 \Omega$ and (1) can be obtained from (2) by supposing a solution of the form $x(t)=x_{1}(t) \sin \Omega t+x_{2}(t) \cos \Omega t$, where $x_{1}$ and $x_{2}$ are assumed to vary sufficiently slowly (cf. the Krylov-Bogoliubov averaging method [9]).

Eqs. (1) and (2) have been discussed extensively by M. Cartwright in [3] and [4]. In particular, she predicted the form of the bifurcation set of (1), namely the set $\mathbf{B}$ of points $(\omega, F) \in R^{2}$ where $X_{(\omega, F)}$ is structurally unstable. In a later paper A. Gillies [5] pointed out that this predicted structure could not be correct and presented an alternative. To do this he adduced arguments from constructions of the vector field for various numerical values of $(\omega, F)$. In this paper we make use of recent developments in the theory of bifurcations of vector fields to provide a more complete characterization of B and thus to confirm Gillies' conjecture.

Under the above correspondence, zeros or singularities of (1) correspond approximately to sinusoidal periodic solutions of (2), while periodic orbits (limit cycles) correspond to non-sinusoidal or almost periodic solutions. Thus the bifurcational structure of $X$, by describing the ways that singularities, periodic orbits, etc. are created and annihi-

\footnotetext{
* Received August 1, 1977. The authors would like to acknowledge particularly the work of F. Takens and M. L. Cartwright. This research was carried out with the financial support of the Science Research Council $(\mathrm{PJH})$ and the Social Science Research Council (DAR).
} 
lated, indicates the ways in which (2) can become 'entrained' or break its entrainment for almost-periodic or 'drift' oscillations [4] as the forcing parameters vary. It also gives information on the existence of homoclinic motions for certain values of the forcing parameters (see Sec. 4.3).

2. The bifurcation set. We say that $\left(\omega_{0}, F_{0}\right) \in R^{2}$ is a bifurcation value of $X$ if $X_{\left(\omega_{0}, F_{0}\right)}$ is a structurally unstable dynamical system (see, e.g., [11]). It follows from the work of Andronov et al. [1] and Peixoto [10] that this will be the case if at least one of the following conditions is violated:

(a) $X_{i \omega, F}$, has a finite number of singularities each of which is hyperbolic ${ }^{1}$;

(b) the closed paths of $X_{(\omega, F)}$ are hyperbolic ${ }^{1}$ periodic orbits;

(c) $X_{i \omega, F}$, has no trajectories which connect saddle points.

The set $\mathbf{B}$ of such points is called the bifurcation set of $X_{(\omega, F)}$. If $\left(\omega_{0}, F_{0}\right) \in \mathbf{B}$, an arbitrarily small perturbation from $\left(\omega_{0}, F_{0}\right)$ can change the qualitative nature of the phase portrait of $X_{i \omega, F}$. Our goal is to describe $(a)$ the structure of $\mathbf{B}$ and $(b)$ the way in which $X_{(\omega, F)}$ changes as we cross B.

The points $(\omega, F)$ where $(a)$ is violated are easily calculated as, for example, in [4]: they fall naturally into two categories.

2.1 Creation and annihilation of singularities; $\mathbf{B}_{s}$. Solving for the zeros of $X$, we find that (1) has two coincident singularities when

$$
v=\frac{F^{4}}{4}-\frac{F^{2}}{27}\left(1+9 \omega^{2}\right)+\frac{\omega^{2}}{27}\left(1+\omega^{2}\right)^{2}=0 .
$$

We denote the set of such points $(\omega, F)$ by $\mathbf{B}_{s}$. In general there is one hyperbolic singularity and one degenerate (i.e. non-hyperbolic) singularity; the exceptional case of a single degenerate singularity at $\mathrm{A}$ :

$$
(\omega, F)=\left(\frac{1}{\sqrt{3}}, \sqrt{\frac{8}{27}}\right)
$$

is discussed separately below. At such a degenerate singularity at least one of the eigenvalues of the linearized equation is zero. When only one is zero, it can be shown (see [12]) that the corresponding singularity is a saddle-node. When $v>0$ there is one singularity which is a node or a focus. When $v<0$ there are three hyperbolic singularities, one of which is a saddle point; of the remaining two, one is always a node and the other may be a node or a focus. Further examination of the eigenvalues allows us to write down the set $\mathbf{B}_{N}$ on which nodes become foci:

$$
F^{2}=\omega\left(1-2 \omega+2 \omega^{2}\right) .
$$

Since the topological type of the orbit structure does not change on this set we shall not generally be concerned with it in the following discussion.

2.2 Creation of limit cycles by a Hopf bifurcation; $\mathbf{B}_{H}$. The other case where $(a)$ is

\footnotetext{
${ }^{1}$ If $x_{0}$ is a singularity of $X_{\omega \omega, F}$, so that $X_{\omega, F}\left(x_{0}\right)=0$, we say that $x_{0}$ is hyperbolic if none of the eigenvalues of the derivative $D X_{\omega . F},\left(x_{0}\right)$ have zero real part. If $\gamma$ is a closed orbit of $X_{\omega, F}$, we say that $\gamma$ is hyperbolic if none of the characteristic multipliers associated with $\gamma$ have unit modulus; this is equivalent to requiring that none of the eigenvalues of the derivative of the associated Poincare map have unit modulus. This implies that hyperbolic singularities and periodic orbits are isolated.
} 
violated occurs when the linearized equation at the singularity has purely imaginary eigenvalues, i.e. when

$$
8 F^{2}=4 \omega^{2}+1 ; \quad|\omega|>\frac{1}{2} .
$$

This curve is tangential to that given by Eq. (3) at the point $0:(\omega, F)=\left(\frac{1}{2}, \frac{1}{2}\right)$. Here the eigenvalues of the degenerate singularity are $(0,0)$ and we have a complex situation which we examine below. The second fixed point does not take part in this bifurcation, remaining a stable node.

Furthermore, we may check that if we cross $\mathbf{B}_{H}$ with non-zero speed (i.e. transversely) the eigenvalues of the degenerate singularity cross the imaginary axis with non-zero speed and on $\mathbf{B}_{H}$ the degenerate singularity is weakly attracting. Thus we may apply a theorem of Hopf [8] on the creation of limit cycles (cf. [12, p. 25]) to prove that an attracting limit cycle appears, surrounding a source.

2.3 The bifurcation set of Cartwright and Gillies. The part of the bifurcation set derived above, which was obtained by Cartwright and Gillies, is shown in Fig. 1. The qualitative
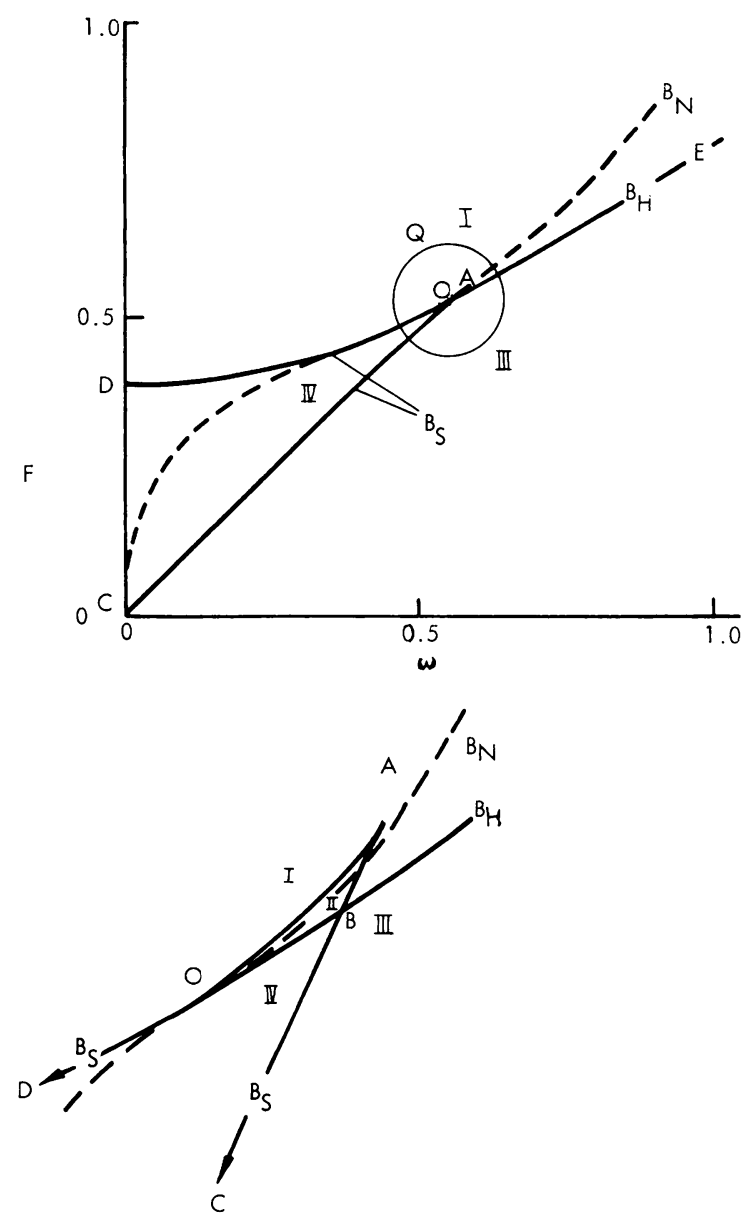

Region $Q$ (enlarged and distorted )

FIG. 1. The bifurcation set of $X_{(\omega, F)}$. 
evolution of the vector field as we cross B transversally, $\omega$ increasing, is shown in Fig. 2. We do not make the distinction between nodes and foci.

The above bifurcations follow from Andronov et al. [1] and Sotomayor [12]. We have not yet considered the nature of $X_{(\omega, F)}$ at $0, A, B=(\sqrt{5} / 4,3 / 4 \sqrt{2})$ or on CB $\subset \mathbf{B}_{s}, C=(0$, $0)$. Before we do this we follow Cartwright and Gillies and note that (see $(b)$ and (e), Fig.2) region IV contains at least two qualitatively different types of vector field and that these are not topologically conjugate. Thus IV must contain bifurcation points.

Cartwright's conjecture, subsequently amended by Gillies in [5], was that we must add a curve $\mathbf{B}_{S C}$ of bifurcation points emanating from 0 and crossing IV to meet the open

(a) CROSSING OPEN SE:GMENT CA

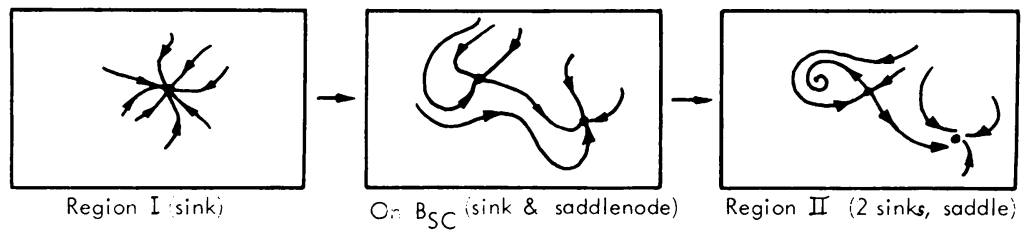

(b) CROSSING OPEN SE GMENT OD

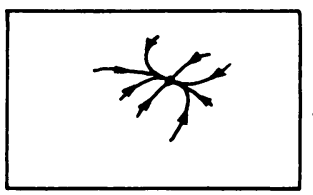

Reçio: I (sink)

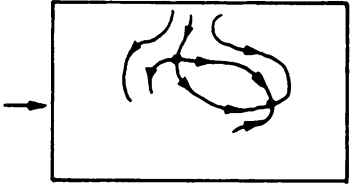

On ${ }_{S C}($ siirk \& saddilenode $)$

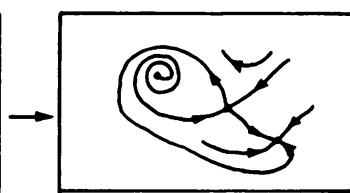

Reçion IV (sirk, source, saddle)

(c) CROSSING OPEN SEGMENT AB

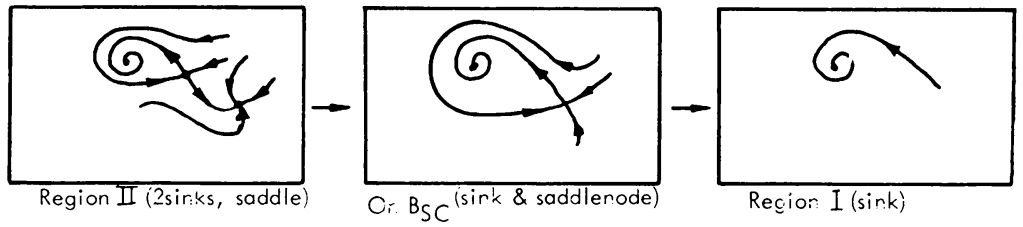

d) CROSSING OPEN SESMENT BE

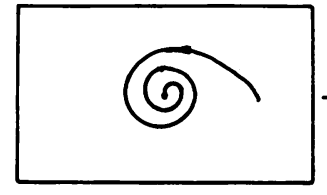

Region I isink)

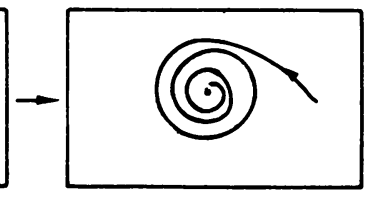

On $\mathrm{B}_{\mathrm{H}}$ (cer:tre)

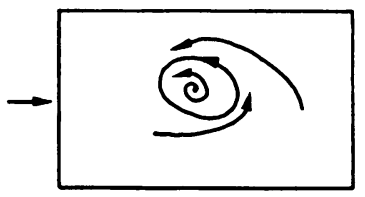

Region III (source \& limit cycle)

(e) CROSSING OPEN SEGMENT OB

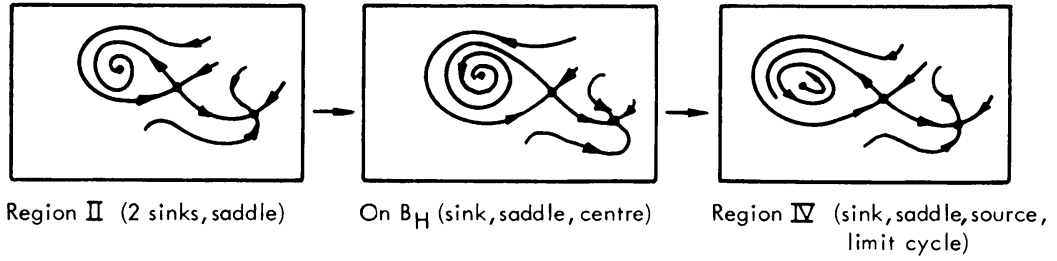

FIG. 2. The vector field $X_{(\omega, F)}$. 
segment $\mathrm{CB}$, such that, for $(\omega, F) \in \mathbf{B}_{S C}, X_{(\omega, F)}$ has a saddle connection, i.e. a point where condition $(c)$ is violated. Since there is only one saddle point this must be a separatrix loop; see Andronov et al. ([1, chapter XI]), where the ways in which such a loop can give rise to a hyperbolic limit cycle are discussed.

The 'global' nature of such a bifurcation prevents a simple derivation of the bifurcation points such as was possible with the 'local' bifurcations analyzed above. Certainly we are not able to obtain an analytic expression for $\mathbf{B}_{S C}$. However, an analysis of the degenerate singularity at 0 allows us to prove the local existence of the conjectured curve and to determine some of its qualitative behavior. First, we deal with the degenerate singularities at $A$ :

and $B$ :

$$
(\omega, F)=\left(\sqrt{\frac{1}{3}}, \sqrt{\frac{8}{27}}\right)
$$

$$
(\sqrt{ } 5 / 4,3 / 4 \sqrt{ } 2) \text {. }
$$

These are both of codimension 2: for the exact definition see Takens [13]; roughly speaking, a codimension- $k$ singularity is one which occurs stably in a $k$-parameter family of vector fields. Since we are concerned with two parameters here, we expect, at worst, codimension-2 singularities.

The point $A . X_{(\omega, F)}$ contains one triple degenerate singularity. Examination of the eigenvalues of the linearized equation indicates that the degeneracy involves two stable nodes (sinks) and a saddle point splitting off a stable node. This is a codimension-2 bifurcation corresponding to the organizing centre of Thom's cusp catastrophe (for more details see the classification in [13]). Note that the two arms DA and CA of $\mathbf{B}_{S}$ meet at $A$ in a two-thirds-power-law cusp.

The point $B$. Here the eigenvalues and analysis indicate that we have two independent codimension- 1 bifurcations taking place simultaneously. In region II, close to $B$ (Figs. 1 and 2 ) there are two sinks (a node and a focus) and a saddle point. At $B=\mathbf{B}_{H} \cap \mathbf{B}_{S}$ the focus undergoes a Hopf bifurcation on $\mathbf{B}_{H}$ and the saddle point and node coalesce on $\mathbf{B}_{S}$.

The qualitative forms of the vector field at $A$ and $B$ are indicated in Sec. 4.1 below.

\section{The singularity at $0:\left(\frac{1}{2}, \frac{1}{2}\right)$.}

3.I The reduction theorem. The reduction procedure described below involves a slight modification of the proof of the normal-form theorem of Takens ([13, Theorem 2.1]; cf. [14]). We discuss it in some detail since it appears to be of quite general use.

Throughout $H^{s}, s \geq 0$, denotes the real vector space of vector fields whose coefficient functions are homogeneous polynomials of degree $s$.

Let $X_{\mu}$ be a given $C^{r}$-vector field (where $r$ is assumed sufficiently large) depending $C^{r}$ smoothly upon parameters $\mu_{1}, \ldots, \mu_{m}$ and such that $X_{0}(0)=0$. Let $A \in H^{1}$ be the linear part of $X_{0}$ and consider the linear map

$$
[A, \cdot]^{k}: \sum_{s=0}^{k} H^{s} \rightarrow \sum_{s=0}^{k} H^{s}
$$

where [.,.] denotes the Lie bracket: if $X=\sum_{i=1}{ }^{n} X^{i}\left(\partial / \partial x_{i}\right)$ and $Y=\sum_{i=1}^{n} Y^{i}\left(\partial / \partial x_{i}\right)$ then

$$
[X, Y]=\sum_{i=1}^{n} \sum_{j=1}^{n}\left(\frac{\partial X^{i}}{\partial x_{j}} Y^{j}-\frac{\partial Y^{i}}{\partial x_{j}} X^{j}\right) \frac{\partial}{\partial x_{i}}
$$


This mapping induces the splitting

$$
\sum_{s=0}^{k} H^{s}=\sum_{s=0}^{k} B^{s}+\sum_{s=0}^{k} G^{s}
$$

where $B^{s}$ is the image of $H^{s}$ under the mapping and $G^{s}$ is some complementary subspace.

Theorem: For $k, l \leq r$ there exists a diffeomorphism $\phi:\left(R^{n}, 0\right) \rightarrow\left(R^{n}, 0\right)$ such that

$$
\phi_{*}\left(X_{\mu}\right)=X_{0} \cdots 0+\sum_{i=1}^{l} \sum_{i_{1}+\cdots+i_{m}=l} \mu_{1}^{i \mathrm{a}} \cdots \mu_{m}{ }^{i_{m}} X_{i_{1}} \cdots i_{m}+R_{l}
$$

where $R_{l}$ is $O(|\mu|) \cdot O\left(|x|^{k+1}\right)+O\left(|\mu|^{l+1}\right)$ and

$$
X_{i_{1} \ldots i_{m}} \in \sum_{s=0}^{k} G^{s} .
$$

Remark: A diffeomorphism $\phi: R^{n}, 0 \rightarrow R^{n}, 0$ acts on the family $X_{\mu}$ by conjugation:

$$
(\phi)_{*}\left(X_{\mu}\right)(x)=D \phi\left(\phi^{-1}(x)\right) . X_{\mu}\left(\phi^{-1}(x)\right),
$$

corresponding to a change of coordinates.

The following result follows from the proof of the theorem:

COROLlary: If $X$ is a vector field of the form

$$
X=X_{1}+X_{2}+\cdots+X_{k}+\left[X_{1}, Y\right]+R_{k}
$$

where $X_{i} \in H^{i}, Y \in H^{k}$ and $R_{k}$ is $0\left(|x|^{k+1}\right)$, then by a change of coordinates we may get $X$ into the form

$$
X=X_{1}+X_{2}+\cdots+X_{k}+R_{k}^{\prime}
$$

where $\mathrm{R}_{k}{ }^{\prime}$ is $0\left(|x|^{k+1}\right)$.

Proof (of Theorem): The proof is by induction on $l$. Suppose we have

$$
X_{\mu}=X_{0} \ldots 0+\sum_{i=1}^{l} \sum_{i_{1}+\cdot+i_{m}=i} \mu_{1}^{i_{1}} \ldots \mu_{m}{ }^{i_{m}} X_{i_{1}} \ldots i_{m}+R_{l}
$$

as in the statement of the theorem; we prove we can replace $l$ by $l+1$. Certainly we can write

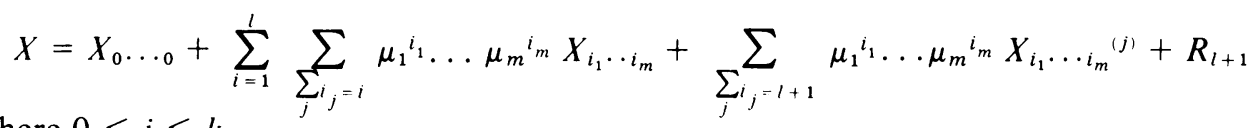

where $0 \leq j \leq k$,

$$
X_{i_{1} \cdots i_{m}}{ }^{(j)} \in \sum_{s=0}^{j} H^{s}
$$

and $R_{l+1}$ is

$$
O\left(|\mu|^{l+1}\right) O\left(|x|^{j+1}\right)+O(|\mu|) \cdot O\left(|x|^{k+1}\right)+O\left(|\mu|^{l+1}\right) .
$$

We prove by induction on $j$ that a change of coordinates will get $X_{i_{1}} \cdots i_{m}^{(j)}$ into $\sum_{s=0}{ }^{j} G^{s}$. Assume

$$
X_{i_{1} \cdots i_{m}}{ }^{(j-1)} \in \sum_{s=0}^{j-1} G^{s}
$$

for all appropriate $i_{1}, \ldots, i_{m}$ and suppose 


$$
X_{i_{1} \cdots i_{m}}{ }^{(j)}-X_{i_{1} \cdots i_{m}}^{(j-1)}=Y_{i_{1} \cdots i_{m}}+Z_{i_{1} \cdots i_{m}}
$$

where

$$
Y_{i_{1} \cdots i_{m}} \in B^{j}, Z_{i_{1} \cdots i_{m}} \in G^{j}
$$

Let $U_{i_{1} \cdots i_{m}} \in H^{j}$ be such that $\left[A, U_{i_{1} \cdots i_{m}}\right]=Y_{i_{1} \cdots i_{m}}$ and let

and

$$
U=\sum_{i_{1} \cdots i_{m}} \mu_{1}^{i_{1}} \ldots \mu_{m}{ }^{i_{m}} U_{i_{1}} \cdots i_{m}
$$

$$
Y=\sum_{i_{1} \cdots i_{m}} \mu_{1}^{i_{1}} \ldots \mu_{m}{ }^{i_{m}} Y_{i_{1}} \ldots i_{m} .
$$

Consider $U$ as a vector field on $R^{n} \times R^{m}$. Then the flow $\phi_{t}:\left(R^{n} \times R^{m}, 0\right) \rightarrow\left(R^{n} \times R^{m}\right.$, 0 ) associated with $U$ is trivial in the $R^{m}$ direction and has the same $(j+l)$-jet in the origin (cf. the Taylor series of order $(j+l)$ ) as the identity. Consequently $X$ and $\left(\phi_{t}\right)_{*}(X)$ differ only in terms of order $j+l$ or greater; in fact, by a term of the form $0\left(|\mu|^{l}\right) .0\left(|x|^{j}\right)+$ $0\left(|\mu|^{I+1}\right)$. Thus

$\left[\left(\phi_{t}\right)_{*}(X), U\right]=[X, U]+O(|\mu|) \cdot O\left(|x|^{j+1}\right)+0\left(|\mu|^{l+1}\right)=[A, U]+R_{l+1, t}=Y+R_{l+1, t}$ where

$$
R_{l+1, t} \text { is } 0(|\mu|) 0\left(|x|^{j+1}\right)+0\left(|\mu|^{l+1}\right) .
$$

From the well-known result on Lie derivatives we have

$$
\left[\left(\phi_{t}\right)_{*}(X), U\right]=\frac{d}{d t}\left(\phi_{t}\right)_{*}(X)
$$

and by Taylor's theorem there exist $A>0$ such that for all $|t|<1$ and all $(x, \mu) \in R^{n} \times$ $R^{m}$ with $\|(x, \mu)\|<1$ we have $\left\|R_{l+1, t}{ }^{\prime}(x, \mu)\right\| \leq A\|(x, \mu)\|$; whence we may integrate the above relations to obtain

$$
\left(\phi_{t}\right)_{*}(X)=X+t Y+R_{l+1, t^{\prime}}
$$

where $R_{l+1, t^{\prime}}$ is $O(|\mu|) O\left(|x|^{j+1}\right)+O(|\mu|) \cdot O\left(|x|^{k+1}\right)+O\left(|\mu|^{l+1}\right)$. If we now consider $\left(\phi_{-1}\right)_{*}(X)$ instead of $X$ we may replace $j$ by $j+1$. We continue in this way until $j=k$, when the inductive step for $l$ is completed.

3.2 The qualitative form of $X_{(1 / 2,1 / 2)}$ at the degenerate singularity. The degenerate (i.e. non-hyperbolic) singularity of $X_{(1 / 2,1 / 2)}$ is at $\left(x_{1}, x_{2}\right)=\left(\frac{1}{2}, \frac{1}{2}\right)$. In this section we carry out the reduction of $X$ and describe this singularity.

If we make the transformation $x_{1}{ }^{\prime}=x_{1}-\frac{1}{2}, x_{2}{ }^{\prime}=x_{2}-\frac{1}{2}, \alpha_{1}=\omega-\frac{1}{2}$ and $\alpha_{2}=\frac{1}{2} \omega-F+\frac{1}{4}$ we have, immediately dropping primes, that

is given by:

$$
X_{\left(\alpha_{1}, \alpha_{2}\right)}=X^{1} \frac{\partial}{\partial x_{1}}+X^{2} \frac{\partial}{\partial x_{2}}
$$

$$
\begin{gathered}
X^{1}=-\frac{1}{2} \alpha_{1}-\left(1+\alpha_{1}\right) x_{2}-x_{1}\left(x_{1}+x_{2}\right)-\left(\frac{1}{2}+x_{1}\right)\left(x_{1}^{2}+x_{2}^{2}\right), \\
X^{2}=\alpha_{2}+\alpha_{1} x_{1}-x_{2}\left(x_{1}+x_{2}\right)-\left(\frac{1}{2}+x_{2}\right)\left(x_{1}{ }^{2}+x_{2}{ }^{2}\right) .
\end{gathered}
$$

For the first reduction we consider $X_{\alpha}$ as an unparameterized system in the sense that we identify it with the vector field

$$
X=X^{1} \frac{\partial}{\partial x_{1}}+X^{2} \frac{\partial}{\partial x_{2}}+0 \cdot \frac{\partial}{\partial \alpha_{1}}+0 \cdot \frac{\partial}{\partial \alpha_{2}}
$$


Applying the corollary to the reduction theorem in this case with

$$
Y=\left(\frac{5}{4} x_{1}^{2}+\frac{1}{2} x_{1} x_{2}\right) \frac{\partial}{\partial x_{1}}+\left(\frac{1}{2} x_{1}^{2}+\frac{3}{2} x_{1} x_{2}\right) \frac{\partial}{\partial x_{2}}
$$

we deduce that by a change of coordinates we may rewrite $X$ so that

$$
\begin{aligned}
& X^{1}=-x_{2}-2 x_{1}{ }^{2}-\alpha_{1}\left(\frac{1}{2}-\frac{5}{4} x_{1}+\frac{3}{4} x_{2}\right)-\frac{1}{2} \alpha_{2} x_{1}+0\left(|x, \alpha|^{3}\right), \\
& X^{2}=-\frac{1}{2} x_{1}^{2}+\alpha_{1}\left(\frac{3}{2} x_{1}+\frac{3}{4} x_{2}\right)+\alpha_{2}\left(1-\frac{3}{2} x_{2}\right)+0\left(|x, \alpha|^{3}\right) .
\end{aligned}
$$

Notice that the coordinate change given by $\phi_{Y,-1}$ is legitimate with respect to the parametrization since $\phi_{Y,-1}$ is trivial in the $\alpha$-direction. We can now apply this technique inductively so that for any natural number $k$ we may write

$$
\begin{aligned}
& X^{1}=-x_{2}-2 \sum_{2}^{k} b_{i} x_{1}{ }^{i}-\alpha_{1}\left(\frac{1}{2}-\frac{5}{4} x_{1}+\frac{3}{4} x_{2}\right)-\frac{1}{2} \alpha_{2} x_{1}+0(|\alpha|) \cdot 0\left(\left|x^{2}\right|\right)+0\left(|x, \alpha|^{k+1}\right), \\
& X^{2}=-\frac{1}{2} \sum_{2}^{k} c_{i} x_{1}{ }^{i}+\alpha_{1}\left(\frac{3}{2} x_{1}+\frac{3}{4} x_{2}\right)+\alpha_{2}\left(1-\frac{3}{2} x_{2}\right)+0(|\alpha|) 0\left(|x|^{2}\right)+0\left(|x, \alpha|^{k+1}\right)
\end{aligned}
$$

after a change of coordinates. Here $c_{2}=b_{2}=1$. A transformation of the form

$$
\left(x_{1}, x_{2}\right) \rightarrow\left(x_{1}+\sum_{2}^{n-1} a_{i} x_{1}{ }^{i}, x_{2}\right)
$$

puts this into the form

$$
\begin{aligned}
& X^{1}=-x_{2}-2 \sum_{2}^{k} b_{i} x_{1}{ }^{i}-\alpha_{1}\left(\frac{1}{2}-\frac{5}{4} x_{1}+\frac{3}{4} x_{2}\right)-\frac{1}{2} \alpha_{2} x_{1}+0(|\alpha|) \cdot 0\left(|x|^{2}\right)+0\left(|x|^{k+1}\right), \\
& X^{2}=-\frac{1}{2} x_{1}{ }^{2}+\alpha_{1}\left(\frac{3}{2} x_{1}+\frac{3}{4} x_{2}\right)+\alpha_{2}\left(1-\frac{3}{2} x_{2}\right)+0(|\alpha|) 0\left(|x|^{2}\right)+0\left(|x|^{k+1}\right) .
\end{aligned}
$$

Now we need to employ the full version of the reduction theorem on

$$
X=X^{1} \frac{\partial}{\partial x_{1}}+X^{2} \frac{\partial}{\partial x_{2}} .
$$

First we note that

$$
\left[-x_{2} \frac{\partial}{\partial x_{1}}, Y\right]=\left(-x_{2} \frac{\partial Y^{1}}{\partial x_{1}}+Y^{2}\right) \frac{\partial}{\partial x_{1}}+\left(-x_{2} \frac{\partial Y^{2}}{\partial x_{1}}\right) \frac{\partial}{\partial x_{2}}
$$

whence for $s \geq 1$ the vector space $H^{s}$ is spanned by $B^{s}, x_{1}^{s} \partial / \partial x_{1}$ and $x_{1}^{s} \partial / \partial x_{2} ; H^{0}$ is spanned by $B^{0}$ and $\partial / \partial x_{2}$. Thus we take $G^{s}$ to be the subspace of $H^{s}$ spanned by $x_{1}^{s} \partial / \partial x_{1}$ and $x_{1}{ }^{s} \partial / \partial x_{2}$ in the case $s \geq 1$ and by $\partial / \partial x_{2}$ when $s=0$.

Applying the reduction theorem, we can get $X$ into a form where

$$
\begin{aligned}
X^{1}= & -x_{2}-2 \sum_{2}^{k} b_{i} x_{1}{ }^{i}-\alpha_{1}\left(\frac{1}{2}-2 x_{1}\right) \\
& -2 \alpha_{2} x_{1}+0(|\alpha|) \cdot 0\left(\left|x_{1}\right|^{2}\right)+0(|\alpha|) 0\left(|x|^{\prime}\right)+0\left(|x|^{k+1}\right), \\
X^{2}= & \alpha_{2}-\frac{1}{2} x_{1}{ }^{2}+\frac{3}{2} \alpha_{1} x_{1}+0(|\alpha|) \cdot 0\left(\left|x_{1}\right|^{2}\right) \cdot+0(|\alpha|) \cdot 0\left(|x|^{\prime}\right)+0\left(|x|^{k+1}\right) .
\end{aligned}
$$

We have started the induction of the proof at $j=1 ; l$ is any large integer. To start off the 
inductive reduction, take

$$
U=\frac{3}{4} \alpha_{1}\left(x_{1} \frac{\partial}{\partial x_{1}}-x_{1} \frac{\partial}{\partial x_{2}}\right)+\frac{3}{2} \alpha_{2} x_{1} \frac{\partial}{\partial x_{2}} .
$$

By a coordinate change of the form $\left(x_{1}, x_{2}\right) \rightarrow\left(x_{1}-\frac{3}{2} \alpha_{1}+0\left(|\alpha|^{2}\right), x_{2}+0(|\alpha|)+\right.$ $\left.0\left(|\alpha|^{i}\right) \cdot 0\left(|x|^{j}\right)\right)$, we may simplify further:

$$
\begin{gathered}
X^{1}=-x_{2}-2 x_{1}{ }^{2}-\left(4 \alpha_{1}+2 \alpha_{2}\right) x_{1}+0(|\alpha|) \cdot 0\left(|x|^{3}\right)+0\left(|x, \alpha|^{k}\right), \\
X^{2}=\alpha_{2}-\frac{1}{2} x_{1}{ }^{2}+0(|\alpha|) \cdot 0\left(|x|^{3}\right)+0\left(|(x, \alpha)|^{k+1}\right) .
\end{gathered}
$$

Taking $\mu_{1}=4 \alpha_{1}+2 \alpha_{2}, \mu_{2}=\alpha_{2}$, ignoring higher terms, and using Borel's theorem (cf. [13, p. 56], we deduce:

Proposition 1: Let $X$ be the vector field defined at the beginning of this section. Then there exists a local diffeomorphism $\phi:\left(R^{2} \times R^{2}, 0\right) \rightarrow\left(R^{2} \times R^{2}, 0\right)$ at 0 such that

$$
\phi_{*}(X)=-\left(x_{2}+2 x_{1}{ }^{2}+\mu_{1} x_{1}\right) \frac{\partial}{\partial x_{1}}-\left(\frac{1}{2} x_{1}{ }^{2}-\mu_{2}\right) \frac{\partial}{\partial x_{2}}+0(|\mu|) \cdot O\left(|x|^{3}\right) .
$$

Moreover, $\phi$ is admissible with respect to the parametrization.

3.3 "Unfolding" of the bifurcation set around 0 . In this section we proceed to determine the bifurcation set near 0 of the parametrized vector field $\tilde{X}=\phi_{*}(X)$ of Proposition 1. This follows Takens [14]. His method seems of general use and for this reason and for the sake of completeness we outline the essential details.

First we note that, by a singular substitution of the form:

$$
x_{1}=t^{2} y_{1}, x_{2}=t^{3} y_{2}, \mu_{1}=t \lambda_{1}, \quad \mu_{2}=t^{4} \lambda_{2} \quad \text { and } t Y=\tilde{X},
$$

we obtain from Eq. (6) a smooth vector field $Y$ given by

$$
\begin{aligned}
& \dot{y}_{1}=-y_{2}-2 t y_{1}{ }^{2}-\lambda_{1} y_{1}+O\left(t^{2}\right), \\
& \dot{y}_{2}=-\frac{1}{2} y_{1}{ }^{2}+\lambda_{2}+O\left(t^{2}\right) .
\end{aligned}
$$

Now note that for $\lambda_{1}=0, t=0$ we have, up to terms in $0\left(t^{2}\right)$, a Hamiltonian system

$$
Y_{0}=-\frac{\partial H}{\partial y_{2}} \frac{\partial}{\partial y_{1}}+\frac{\partial H}{\partial y_{1}} \frac{\partial}{\partial y_{2}},
$$

where the Hamiltonian $H$ is given by

$$
H=\frac{1}{2} y_{2}{ }^{2}+\lambda_{2} y_{1}-\frac{1}{6} y_{1}{ }^{3} .
$$

Thus we consider the case where we have a two-parameter family $Y_{\left(\eta_{1}, \eta_{2}\right)}$ (we shall take $\eta_{1}$ $=\lambda_{1}, \eta_{2}=\mathrm{t}$ and fix $\left.\lambda_{2}\right)$ of vector fields where $Y_{(0,0)}$ is a non-degenerate Hamiltonian system with Hamiltonian $H$.

The theorem we apply was first assembled in [14, p. 23]. The basic idea is simple. If we fix $\lambda_{2}=1$ so that $H$ has some compact level curves, the level curves of $H$, and hence the trajectories of

$$
Y_{0}=-\frac{\partial H}{\partial y_{2}} \frac{\partial}{\partial y_{1}}+\frac{\partial H}{\partial y_{1}} \frac{\partial}{\partial y_{2}},
$$

are as in Fig. 3. In this very degenerate phase portrait one can see the ingredients of the degeneracies we expect in $X$, for example a saddle connection. The question is to determine how these degeneracies are maintained when we perturb $Y$ away from $Y_{0}$. To answer 


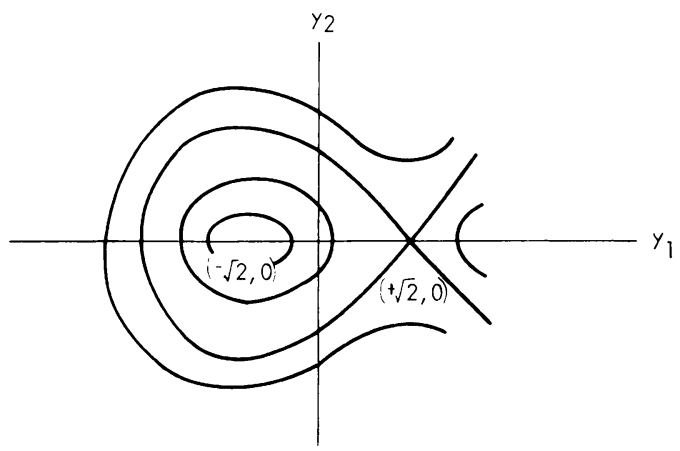

FIG. 3. The Hamiltonian system $Y_{0}$.

this we consider the function $f: R^{2} \times S^{1} \rightarrow R^{1}$ defined by

$$
f(y, \theta)=\lim _{r \rightarrow 0} \frac{1}{r} \quad \operatorname{div} Y_{i r \cos \theta, r \sin \theta i}(y) .
$$

Using $\int$ we can partition $S^{1}$ into regular and non-regular values and determine which of the non-regular values are regular bifurcation values; the reader is referred to [14, $\$ 5]$ for the exact definitions. Theorems 5.4 and 5.6 of [14] give the local bifurcational behavior at 0 of $Y$ in directions near regular values and regular bifurcation values. In particular, if $\gamma:[0,1)$ $\rightarrow R^{2}$ is a differentiable curve starting at $0 \in R^{2}$ and if $\theta$, the initial angle of $\gamma$, is a regular value then there exists $\epsilon>0$ such that $\eta \in \gamma((0, \epsilon))$ implies that $Y_{\eta}$ is structurally stable. If $\theta$ is a regular bifurcational value there exists such a curve $\gamma$ with initial angle $\theta$ such that the topological type of the phase portrait of $Y_{\eta}$ changes as $\eta$ crosses $\gamma$ transversely, in one of the codimension-one ways described by Sotomayor [12]. Details of the ways in which the fields can change are given in [14]; the proofs of the results in $\S 5$ there are essentially contained in Andronov et al. [1], Sotomayor [12] and Takens [13].

Our Hamiltonian is $H=\frac{1}{2} y_{2}{ }^{2}+y_{1}-\frac{1}{6} y_{1}{ }^{3}$. This is a Morse function with two critical points, a minimum at $(-, 2,0)$ and a saddle point at $(, 2,0)$. The function $f$ is given by

$$
f\left(y_{1}, y_{2}, \theta\right)=-4 y_{1} \sin \theta-\cos \theta
$$

in this case, and a straightforward computation shows that all $\theta \in S^{1}$ are regular except for two values $\theta^{h}$ and $\theta^{s}$. A slightly more complicated calculation shows that both of these are regular bifurcation values.

The direction $\theta^{h}=\tan ^{-1}(1 / 4,2)$ corresponds to a curve $\gamma^{h}$ such that when $\eta$ crosses $\gamma^{h}$ a sink of $Y_{\eta}$ throws off an attracting limit cycle (Hopf bifurcation); the calculation here follows as in Takens' example [14] though the reader should observe that this contains several minor mistakes.

We now turn to the bifurcation value $\theta^{s}$. This is a solution of the equation

$$
\int f\left(y_{1}, y_{2}, \theta\right) d y_{1} d y_{2}=0
$$

where the integral is over the area enclosed by the closed component of the level curve of $H$ through the saddle point $(, 2,0)$. It follows from a calculation using standard methods that the only solution is $\theta^{s}=\tan ^{-1}(7 / 20,2)$. This value turns out to be a regular bifurcational value and moreover one can check that if the associated curve is $\gamma^{s}$ then the 
bifurcation occurring as $\eta$ crosses $\gamma^{s}$ involves a source surrounded by an attracting limit cycle which vanishes in the saddle separatrix connection, leaving a source and saddle point alone (cf. Sotomayor $[12, \S 4]$ ). Note that $\theta^{h}<\theta^{s}$.

Finally, so far as bifurcations of $Y$ are concerned, we note that if $\eta_{1}<0$ then $Y_{\eta}, \eta=$ $\left(\eta_{1}, \eta_{2}\right)=\left(\lambda_{1}, t\right)$, has no singularities and that two singular points are born via a saddle node as $\eta$ crosses the $\eta_{2}$-axis.

Thus we have three curves of bifurcation points emanating from the origin whose initial directions we know. This is enough to determine the local behavior of their images at the origin of $\mu$-space under the mapping $\left(\eta_{1}, \eta_{2}\right)=\left(\lambda_{1}, t\right) \mapsto\left(t \lambda_{1}, t^{4}\right)$. Following this through and transforming back to $X_{(\omega, F)}$ we have:

Proposition 2: The bifurcation set of $X_{(\omega, F)}$ near $0:(\omega, F)=\left(\frac{1}{2}, \frac{1}{2}\right)$ consists of three curves $\mathbf{B}_{S}, \mathbf{B}_{H}$ and $\mathbf{B}_{S C}$ as shown in Fig. 4. Along $\mathbf{B}_{S}$ we have the birth of singular points, along $\mathbf{B}_{H}$ a $\mathbf{H}$ opf bifurcation and along $\mathbf{B}_{S C}$ a saddle connection.

The evolution of the phase portrait as we cross these curves is shown in Figs. 2, 5 and 7 below.

Proof. It remains only to check that there are no closed integral curves of $X_{(\omega, F)}$ whose radii grow infinitely large as $(\omega, F) \rightarrow\left(\frac{1}{2}, \frac{1}{2}\right)$. Such a cycle, if it existed, would have to enclose only the sink which remains hyperbolic at 0 . Otherwise it would have to pass between two of the singularities involved in the bifurcations analyzed above and our analysis would have picked it up. However, in region I the stable manifold of the sink consists of the whole plane and hence no such integral curve can exist.

Notice that our new calculation for points where we have the birth of singular points and Hopf bifurcations agrees with the analysis of Sec. 2. The analysis in this section however, provides a proof of the existence of the curve $\mathbf{B}_{S C}$ of saddle connections, at least in a neighborhood of 0 , and allows us to calculate its initial direction below and tangent to $\mathbf{B}_{H}$ and $\mathbf{B}_{S}$.

3.4 The global set $\mathbf{B}_{S C}$. The results of the last section only give $\mathbf{B}_{S C}$ in a neighborhood of 0 . We now extend this result to show that $\mathbf{B}_{S C}$ "crosses" the whole of the region $\mathbf{\Sigma}=$ $\left\{(\omega, F) \in R^{2} \mid \omega, F>0\right.$ and $X_{i \omega, F)}$ has a saddle point $\}$ (region IV).

First, however, we note that, away from $0, \mathbf{B}_{S C}$ need not be a curve although "generically" this will be true and since the equation we are studying is drawn from a physical system we should expect this to be so. Note that it is theoretically possible to check if $\mathbf{B}_{S C}$ is

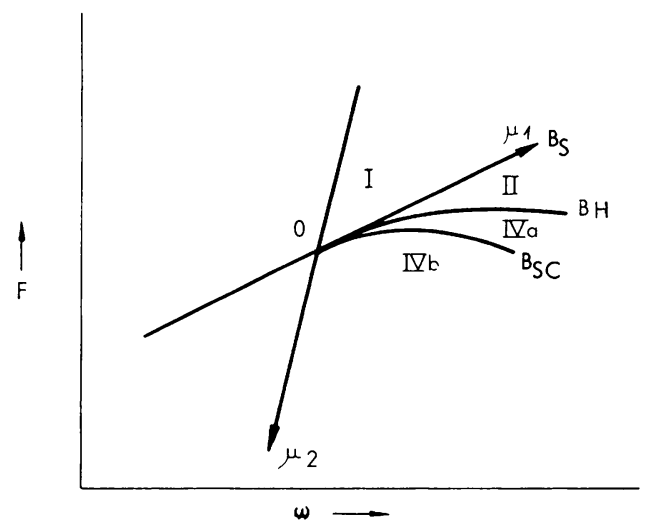

FIG. 4. The bifurcation set at 0 . 
a curve, for if $f$ is the function described in $\$ 4$ of Sotomayor [12] and the derivative of the real-valued function $g:(\omega, F) \rightarrow f\left(X_{(\omega, F)}\right)$ exists and is non-zero for all $(\omega, F) \in \mathbf{B}_{S C}$, then $\mathbf{B}_{S C}$ is a one-dimension submanifold of $\Sigma$. However, such a calculation looks formidable and we have not completed it.

We now describe what we mean by saying $\mathbf{B}_{S C}$ crosses $\mathbf{\Sigma}$. Foliate $\mathbf{\Sigma}$ by a family of noninteresecting curves which start on $O B$, and end on $C D$ (see Fig. 1) and which are transverse to the lines $F=$ constant.

We assume henceforth that for all $(\omega, F) \in \mathbf{\Sigma}, X_{(\omega, F)}$ has only hyperbolic closed trajectories and that the $\alpha$ and $\omega$ limit sets of every trajectory of $X$ are singular points, closed trajectories or saddle separatrix loops. Also we note that if $q$ is the saddle point of $X_{(\omega, F)}$ then $\operatorname{div} X_{(\omega, F)}(q) \neq 0$ so that any saddle separatrix loop is simple.

Then if $M$ is the set of $\omega \in R$ such that $\left(\omega, \frac{1}{2}\right) \in \mathbf{\Sigma}$ and $N$ consists of those $\omega \in M$ where the curve $\gamma$ through $\left(\omega, \frac{1}{2}\right)$ contains a point $\left(\omega^{\prime} F^{\prime}\right)$ such that $X_{\left(\omega^{\prime}, F^{\prime}\right)}$ has a saddle separatrix loop which is simple, then $N$ is open (cf. Sotomayor $[14, \S 4]$ ) and non-empty (Proposition 2 ). Let $\omega^{*}$ belong to the closure of $N$ in $M$. If $\left(\omega^{*}, \frac{1}{2}\right) \in \mathbf{\Sigma}$ the curve $\gamma^{*}$ through $\left(\omega^{*}, \frac{1}{2}\right)$ contains a saddle connection bifurcation point which by the above must consist of a simple loop, whence $\omega^{*} \in N$. Thus $N$ is open and closed in $M$, and non-empty, implying $N$ $=M$ : we thus have:

The bifurcation set of $X_{(\omega, F)}$ contains a curve $\mathbf{B}_{S C}$ which is described in a neighborhood of 0: $(\omega, F)=\left(\frac{1}{2}, \frac{1}{2}\right)$ in Proposition 2 and which crosses every member of a curve foliation of the type described above.

Notice that the above does not prove that $\mathbf{B}_{S C}$ ends on the interior of $A C$. However, if we assume that $\mathbf{B}_{S C}$ is a curve and consists of the only bifurcation points in $\mathbf{\Sigma}$, then it would seem to follow from Gillies' arguments and drawings [5, p. 161] that $\mathbf{B}_{S C}$ meets $A C$ at a point $S$ as described there (cf. Fig. 7 and discussion in Sec. 4.2). Moreover, the argument. ending 4.2 tells us that $\mathbf{B}_{S C}$ cannot approach $C$.

4. The remainder of the bifurcation set: conclusion. We can now complete the discussion of the bifurcation set $\mathbf{B}$ and the associated vector fields $X_{(\omega, F)}$ begun in Sec. 2 . We first indicate the structure of $\mathbf{B}$ where it is established and then proceed to conjecture the remaining structure.

(a)

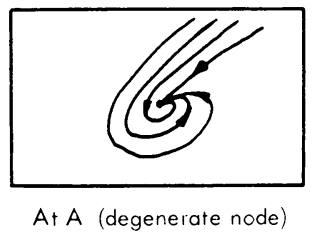

(b)

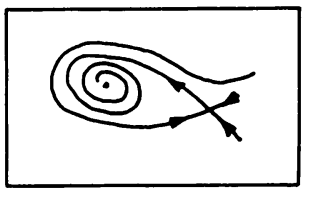

At $B$ (centre \& saddle rode)
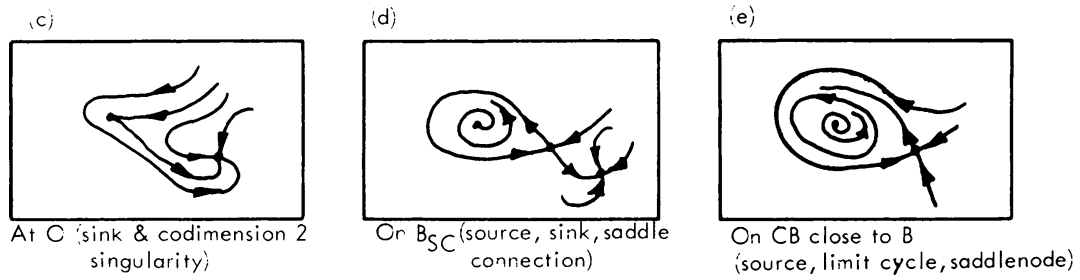

FIG. 5. The remainder of the vector field $X_{\omega, \boldsymbol{F}}$ established. 


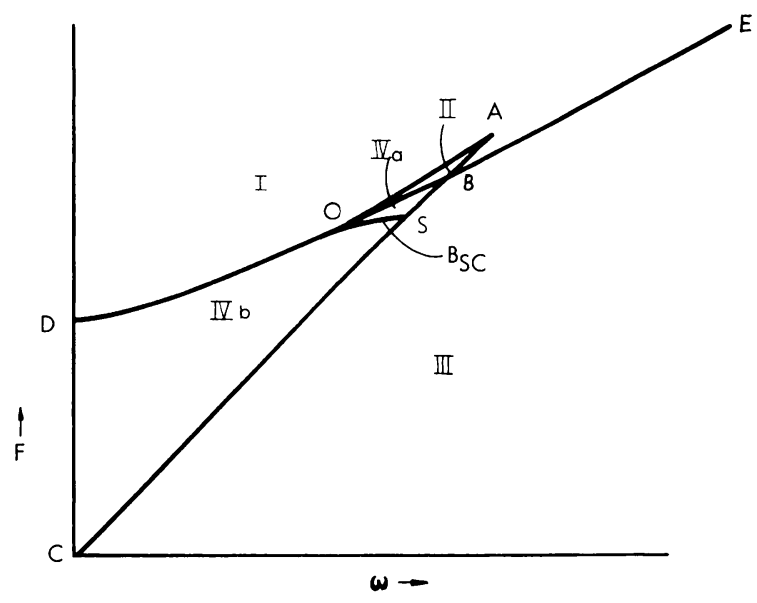

FIG. 6. The complete bifurcation set: conjectured.

4.1 Remaining portions of $\mathrm{B}$ and associated vector fields: established. The established structure of the bifurcation set in the neighborhood of 0 is described in Proposition 2 and Fig. 4. In Fig. 5 we indicate the form of $X_{(\omega, F)}$ at 0 , on $\mathbf{B}_{S C}$, at the points $A$ and $B$ and also on $C B \subset \mathrm{B}_{S}$ close to $B$. In the latter we agree with Cartwright and Gillies, since at $B$ the Hopf-bifurcation and saddle-node coalescence occur simultaneously but independently. As we move transversely across $\mathbf{B}_{H}$, the limit cycle grows as the square root of the distance from $\mathbf{B}_{H}$. The conjectured point $S$ on $C B$ where it meets the saddle node is discussed below.

The set $\mathbf{B}_{S C}$ divides region IV into two regions (IVa and IVb). In IVa, $X_{(\omega, F)}$ has the form indicated in the right-hand picture of $2(e)$ and in IVb that indicated in the right-hand picture of $2(b)$.

4.2 Remaining portions of $\mathrm{B}$ and associated vector fields: conjectures and conclusion. Henceforth we conjecture that $X_{(\omega, F)}$ is a generic family of vector fields, implying that $\mathbf{B}_{S C}$ is a curve. We complete our discussion of the bifurcations by considering $C B \subset \mathbf{B}_{s}$ not close to $B$. On $C B$ near $\mathbf{B}$ the vector field has the form indicated in Fig. $5(e)$. As we move down $C B$ the limit cycle grows, just as it would for a nearby path in $\mathbf{\Sigma}$, until we reach $S$ $\in \mathbf{B}_{s} \cap \mathbf{B}_{S C}$ (see Fig. 6) where a saddle node connection is formed as in Fig. 7(a). We conjecture with Gillies that $S$ is a point close to $B$. The bifurcation occuring at $S$ is of codimension two since the connection here corresponds to the saddle connections of the nearby fields. We would expect that this is not true of the fields corresponding to points further down $C B$ whose form is indicated in $7(b)$. These possess the property that the $\alpha$ separatrix of the indicated saddle node has the saddle node itself as $\omega$ limit, the whole curve being diffeomorphic to the circle. The annihilation of this saddle node leaves a closed orbit and this bifurcation is of codimension one, as we would expect, (cf. Andronov [1, Chapter XII]).

A study of the nature of the vector field at $C:(\omega, F)=(0,0)$ gives us some more information. The vector field here is

$$
X_{(0,0)}=x_{1}\left(1-x_{1}^{2}-x_{2}^{2}\right) \frac{\partial}{\partial x_{1}}+x_{2}\left(1-x_{1}^{2}-x_{2}^{2}\right) \frac{\partial}{\partial x_{2}},
$$


(a)

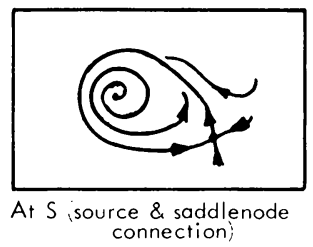

(b)

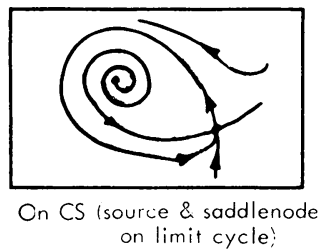

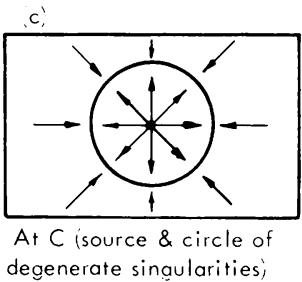

FIG. 7. The remainder of the vector field $X_{u^{\prime}, F}$ established and conjectured.

or, in polar coordinates,

$$
\tilde{X}_{(0,0)}=r\left(1-r^{2}\right) \frac{\partial}{\partial r}+0 \cdot \frac{\partial}{\partial \theta},
$$

from which we immediately see that the origin at $(0,0)$ is a source and that the unit circle, $r=1$, consists of degenerate singular points; the vector field is radial as shown in Fig. 7(c). Using the work of Hirsch, Pugh and Shub [7], we note that this circle is a 2-normally hyperbolic manifold and is therefore preserved as an invariant manifold under small perturbations of the parameters $(\omega, F)$ : i.e., a closed invariant curve diffeomorphic to a circle will continue to surround the source. The conjectured forms of the vector field in IV $b$, and $C S$, and in region III (Figs. $2(b), 7(b)$ and $2(d))$ are consistent with this. Also, this rules out the possibility that $X_{(\omega, F)}$ has a saddle connection for $(\omega, F)$ near $(0,0)$.

4.3 Physical implications. The forced van der Pol equation (2) first arose in studies of radio valves (see Cartwright [4] for background). Today such applications are less relevant but the equation occurs widely in stability problems in aeroelasticity and structural mechanics and in chemical reaction-diffusion processes.

The correspondence between the critical elements (sinks, closed orbits, etc.) of (1) and solutions of (2) may now be used to discuss the physical implications of the behavior detected above. Recall that singularities of (1) correspond to entrained (sinusoidal) periodic solutions of (2) and that closed orbits of (1) correspond to non-sinusoidal (almost) periodic drift solutions. Referring to Fig. 6, then, in region I and IVb the single attractor of (1) is the sink and thus these regions will be characterized by entrained behavior. In region II there are two sinks and a competition of attractors ensued with jumps between the entrained regions on $O A$ and $O B$. In region III the sole attractor is the closed orbit and drift oscillations will be observed. In region IVa, however, we have a sink in addition to the closed orbit and (2) can therefore exhibit either entrained or drift oscillations. Crossing $O B<B_{H}$ the closed orbit evolves continuously from one of the sinks of II and the onset of drift oscillations is thus gradual (cf. Fig. 2(e)), while the saddle connection on $\mathbf{B}_{\mathrm{SC}}$ involves the annihilation of the closed orbit and thus a sudden suppression of drift oscillations and a jump to entrained oscillations can occur. There is an important distinction between such strong bifurcations and weak ones, such as that on $\mathbf{B}_{H}$ (cf. Arnold [2]). In the former case, where attractors are annihilated, relatively sudden jumps in behavior result which clearly 
may have important consequences in control applications. In the latter case there is a continuous evolution of attractors without the characteristic hysteresis behavior associated with strong bifurcations. The saddle-node annihilation occurring on $C S$ is similarly a strong bifurcation and thus the breaking of entrainment on $C S \subset \mathbf{B}_{S}$ is significantly different from that on $\mathbf{B}_{H}$, where the natural oscillation component grows from zero rather than appearing suddenly with finite amplitude (cf. Figs. 2(d) and 7(b)). The strong bifurcations on $O A$ and $B A$ are not as important, however, since the singularities are all relatively close and the jumps occur between two regions of entrained oscillations.

In Sec. 1 we noted that the correspondence between solutions of (1) and (2) is approximate. The question remains whether this approximation can be made qualitatively exact. The forced van der Pol oscillator (2) can be regarded as a first-order system on $R^{2} \times$ $S^{1}$ and the qualitative nature of this system is completely determined by the Poincare diffeomorphism $P: R^{2} \rightarrow R^{2}(P$ is the time $2 \pi / \Omega$ flow map). The Taylor series of $P$ at 0 is differentiably conjugate to the Taylor series of the time 1 map of some vector field $Y$ on $\mathrm{R}^{2}$ [14]. An obvious conjecture is that $Y$ is essentially the averaged equation (1) at 0 . If this is so, it appears to follow from the work of Afraimovic and Sil'nikov [15] that, for some parameter values near $\mathbf{B}_{s c}$, (2) will possess a solenoid (Zeeman [16]; cf. Smale's horseshoe [11]), and hence an infinite number of periodic orbits. This is the phenomenon observed by Cartwright and Littlewood in [17], and studied, in a modified context, by Levinson [18], the work which motivated Smale's construction of the horseshoe. Our present work is aimed towards a clarification of this remark.

\section{REFERENCES}

[1] A. A. Andronov, E. A. Leontovich, I. I. Gordon and A. G. Maier, Theory of bifurcations of dynamic systems in the plane (translated from the original Russian), Israel Program of Scientific Translations, Jerusalem, 1971

[2] V. I. Arnol'd, Lectures on bifurcations in versal families, Russian Math. Surveys 27, 54-123 (1972)

[3] M. L. Cartwright Forced oscillations in non-linear systems: contributions to the theory of nonlinear oscillations, Annals Math. Studies No. 20, 1, 149-241 (1950)

[4] M. L. Cartwright, Forced oscillations in nearly-sinusoidal systems, J. Inst. Elec. Eng. 95, 88 (1948)

[5] A. W. Gillies, On the transformation of singularities and limit cycles of the variational equations of van der Pol, Quart. J. Mech. Appl. Math. 7, 152-167 (1954)

[6] C. Hayashi, Nonlinear oscillations in physical systems, McGraw Hill, 1964

[7] M. W. Hirsch, C. C. Pugh and M. Shub, Invariant manifolds, Bull. Am. Math. Soc. 76, 1015-1019 (1970)

[8] E. Hopf, Abzweigung einer periodischen Lösung eines Differentialsystems, Akad. Wiss. Leipzig 94, 1-22 (1942)

[9] N. Krylov and N. N. Bogoliubov, Introduction to Non-linear Mechanics translated by S. Lefschetz, Annals of Math. Studies 11, Princeton, 1947

[10] M. C. Peixoto and M. M. Peixoto, Structural stability in the plane with enlarged boundary conditions, An. Acad. Brasil. Sci. 31, 135-160 (1959)

[11] S. Smale, Differentiable dynamical systems, Bull. Amer. Math Soc. 73, 747-817 (1967)

[12] J. Sotomayor, Generic one-parameter families of vector fields on two-dimensional manifolds, Publ. I.H.E.S. 43, 5-46 (1974)

[13] F. Takens, Singularities of vector fields, Publ. I.H.E.S. 43 47-100 (1974)

[14] F. Takens, Forced oscillations and bifurcations, in Applications of global analysis, Comm. 3 of the Math. Institute, Rijksuniversiteit Utrecht, 1974

[15] V. S. Afraimovic and L. P. Sil'nikov, On small periodic perturbations of autonomous systems, Soviet Math. Dokl. 15, 206-211 (1974)

[16] E. C. Zeeman, Morse inequalities for diffeomorphisms with shoes and flows with solenoids, in Dynamical systems-Warwick 1974, ed. A. Manning, Lecture Notes in Mathematics No. 468, Springer-Verlag, 1975

[17] M. L. Cartwright and J. E. Littlewood, On non-linear differential equations of the second order: I. The equation $\ddot{y}+k\left(1-y^{2}\right) \dot{y}+y=b \lambda k \cos (\lambda t+a), k$ large, J. London Math. Soc. 20, 180-189 (1945)

[18] N. Levinson, A second order differential equation with singular solutions, Annals of Math. 50, 127-153 (1949) 